\title{
Capítulo 1 \\ El primer viaje panamericano
}

Durante la primera mitad del siglo diecinueve Estados Unidos se dedicó a mirar hacia dentro, a explorar su territorio y a desarrollar su economía. Hacia mediados de siglo el país ya alcanzaba los límites de sus propias fronteras, dándose cuenta de que el proceso de expansión debía volverse otro. Entonces la mirada estadounidense se posó sobre la frontera con México y vino la guerra (18461848) que terminó con el 30 por ciento del país latinoamericano siendo anexado por Estados Unidos. Alrededor de los mismos años, grupos de filibusteros incursionaban en Centro América y el Caribe, pero, oficialmente, la mirada sobre América Latina como región era una de simpatía desapegada, sin intenciones de mayores relaciones ni culturales ni económicas. Esto cambió alrededor de 1880, cuando Estados Unidos se vio excedido por su propia producción durante la recesión que lo golpeó entre 1882 y 1885. América Latina comenzó entonces a configurarse como una posibilidad para resolver lucrativamente el problema de la sobreproducción. Es decir, pasó a ser vista como un territorio a conquistar económicamente y en el cual descargar el exceso de la industrialización. Para estos años, la elite latinoamericana ya se había apropiado del término "Latinoamérica" para nombrar a la región, usando el concepto acuñado en Francia, para acercarse más al país europeo y marcar distancia con Estados Unidos, con esa América del Norte a la que veía con demasiadas ansias de avanzar hacia el sur'. La visión de Latinoamérica como mercados hacia los que expandirse está definitivamente presente ya para 1881, cuando el entonces secretario de Estado, James G. Blaine, se inspiró en la doctrina Monroe para imaginar una conferencia de Estados americanos con el fin de discutir disputas territoriales entre México y Guatemala, y Chile y Perúz. Blaine veía a Estados Unidos como árbitro, como guía, pero no solo de esos conflictos en particular sino de todo el hemisferio. Joseph Smith cuenta en "The First Conference of American States" que Blaine:

1 Ver Gobat (2013).

2 La llamada doctrina Monroe, de 1823, proclamaba que desde ese minuto el hemisferio occidental no sería considerado "as subjects for future colonization by any European power" y que cualquier asomo de imperialismo europeo en la región sería considerado una amenaza a la paz y seguridad de la región. No obstante, no sería hasta fines del siglo XIX que Estados Unidos tendría el suficiente poder militar para hacer respetar la doctrina con la que reclamaba su influencia en el hemisferio. Para más sobre esto ver Nester (2013).

2 Open Access. (C) 2021 Soledad Marambio, published by De Gruyter. (c) BY-NC-ND This work is licensed under the Creative Commons Attribution-NonCommercial-NoDerivatives 4.0 International License. 
Insisted that the conference might have fostered a closer relationship between the United States and Latin America and allowed a chance for the United States to exert a "moral influence" that would be "beneficent and far-reaching" in promoting peace and civilization throughout the hemisphere (Smith 2000: 21).

La conferencia de 1881 no prosperó ${ }^{3}$, pero con su propuesta Blaine se ungió como el gran promotor del panamericanismo, presentado entonces como el llamado a la unión y cooperación de los países del hemisferio, pero que, como ya se planteó en la introducción de este libro, entiendo aquí como el movimiento que nace a fines del siglo diecinueve en el seno de un Estados Unidos que busca expandir su influencia económica y política hacia Latinoamérica. La inspiración que Blaine encuentra en la doctrina Monroe es clave para entender uno de los motores internos del panamericanismo: la idea de alejar toda influencia europea de territorio latinoamericano, haciendo a la región más susceptible de conquistar económicamente y de influir políticamente por una potencia interna creciente. Esta idea se pondrá en evidencia especialmente en tiempos de crisis, como durante la Primera y la Segunda Guerra Mundial, cuando Estados Unidos, a través de su retórica panamericanista tratará primero de asentar su influencia y después, en la Segunda Guerra, de alejar la amenaza de la influencia nazi en la región.

Antes de seguir avanzando es necesario hablar del primer panamericanismo, el pensado por Simón Bolívar y cuyas ideas sería reapropiadas, torcidas, por el movimiento que luego enarbolaría Blaine. En el Congreso de Panamá de 1826 el Libertador venezolano propuso una federación de Estados americanos compuesta solo por las antiguas colonias españolas ${ }^{4}$. Para él, la particularidad y originalidad de la América que formaban estas naciones era su ser mestizo, que se contraponía a la esencia blanca de Norteamérica. Esta diferencia, más las producidas por la marca del catolicismo y del protestantismo, respectivamente, volvía incompatibles a las dos regiones. Además, según Sara Castro-Klarén, Bolívar imaginaba la Pan-América como una forma de contención contra el la-

3 Cuenta Smith que la propuesta de Blaine fue hecha sin el respaldo de los países involucrados en los conflictos a arbitrar y que, además, por ser anunciada muy poco antes de que Blaine tuviera que dejar su puesto, fue vista como una maniobra de Blaine para asegurarse una cuota de poder personal (Smith 2000: 20-21).

4 Estados Unidos habría recibido una invitación al Congreso de Panamá sin la venia de Bolívar. Finalmente, los dos delegados estadounidenses no asistieron a la reunión, por motivos fortuitos: uno de ellos murió camino a Panamá y el otro no consiguió que se aprobaran a tiempo los gastos requeridos para hacer el viaje. 
tente imperialismo de Estados Unidos ${ }^{5}$. La reapropiación, entonces, del término de panamericanismo que Estados Unidos efectúa en 1881 requiere de, como dice Castro-Klarén, “a violent resemantization of Bolívar's concept” (Castro-Klarén 2003: 26). En otras palabras, podríamos decir que lo que hace Estados Unidos es su propia lectura y traducción del término bolivariano ${ }^{6}$. Blaine y quienes modulan con él este panamericanismo liderado por Estados Unidos violentan el concepto bolivariano hasta transformarlo en una antítesis de sí mismo. Lawrence Venuti, quien adscribe a la mirada sobre la traducción como un espacio en el que confluyen fuerzas lingüísticas, culturales, económicas e ideológicas, dice que el traductor "always exercises a choice concerning the degree and direction of the violence at work in any translating" (Venuti 2008: 15). Quiero extender esta idea a la forma en que se manipula el término de panamericanismo en el escenario que exploro ${ }^{7}$. Tanto Blaine y sus colaboradores más cercanos, como William Curtis, un periodista de Chicago en quien recayó la mayor parte del esfuerzo administrativo de la Conferencia Panamericana, conocían las ideas de Bolívar y sabían cómo querían usarlas. Siguiendo su propia agenda ideológica se reapropiaron el concepto bolivariano y lo tradujeron, torciéndolo en su paso de una lengua a otra, de una cultura a otra, de una época y de un territorio específico a otro. Sabían el grado de violencia que ponían sobre el término, también tenían muy clara la dirección en la que se ejercía.

Sylvia Molloy dice que toda traducción implica una descontextualización, una extracción desde un contexto de origen para luego colocar el término desplazado en una ubicación inesperada ${ }^{8}$. Podemos pensar esa descontextualización y ese reubicar un término o una idea, como en la violencia de la que habla Venuti. La reubicación, en el caso del término panamericanismo, es la agenda imperialista de Estados Unidos, que busca ampliar su influencia sobre Latinoamérica y que tiene como hito inaugural la Conferencia Panamericana que se

5 Castro-Klarén recuerda que Bolívar siempre habló y escribió sobre "América”, sin la necesidad de hacer la distinción que haría años después José Martí al hablar de "Nuestra América”. Para Bolívar, América “. . . Referred to an indivisible territory, and inaugurated an indivisible, shared social and symbolic order” (Castro Klarén 2003: 33). Este territorio indivisible era, como ya se dijo, el de las antiguas colonias españolas, es decir, una tierra mestiza, fuertemente marcada por el catolicismo.

6 Como dice Patricia Willson siguiendo a Borges: "una traducción es la escritura de una lectura” (Willson 2001).

7 Si bien en The Translator's Invisibility Venuti se refiere específicamente a los traductores literarios independientes (freelance), que trabajan bajo ciertas condiciones precarias y específicas, me parece que la idea se puede extender aquí a estos traductores ocasionales, Blaine y Curtis.

8 Molloy habla de la traducción como un objet trouvé en Molloy (1998). 
llevó a cabo entre 1889 y 1890. Casi una década después de su primer intento, Blaine por fin ve que su insistencia con el tema del panamericanismo va a dar frutos. El Senado aprueba la conferencia y comienzan los preparativos y los anuncios. Smith dice que la retórica de Bolívar inspira la de Blaine y las ideas del Libertador son revestidas, violentamente en este contexto imprevisto, y presentadas en la convocatoria a la conferencia. Meses antes de que comience la reunión de los Estados, se suceden las notas de prensa anticipando la ocasión. Una de ellas, publicada como anónima, pero en realidad escrita por Curtis, explica que "the primary purpose of this congress is international fellowship, as desired by Bolivar” (Smith 2000: 24) ${ }^{9}$. La gran idea bolivariana de una unión de Estados americanos formada por antiguas colonias españolas termina entonces convertida en un amplio llamado a la amistad internacional hemisférica, sin exclusiones. La idea de un hemisferio unido, fuerte, comienza a enarbolarse como si fuera una herencia del Libertador, quien nunca la pensó viable ni recomendable. Al mismo tiempo que esta retórica de la fraternidad se promueve incansablemente en la prensa y con los países invitados a la conferencia, en el interior de la máquina política de Estados Unidos se habla claramente de la necesidad de expandir la influencia económica y política en Latinoamérica. En 1888 el senador demócrata Richard Townshend expuso así su idea de colaboración regional:

The largest and most inviting field for enterprise on Earth exists in the countries south of us in the American continent. Their natural resources are incalculably valuable, and their trade and commerce are capable of immense extension . . . We should not only have a larger share of that trade than any country but we should be able to control most of it. It is the only great market left for our surplus products (Smith 2000: 22).

Blaine se suma a la mirada expansionista y declara que su idea de panamericanismo siempre tuvo en consideración el crecimiento de los mercados. Dicho esto, se pone a la cabeza de la organización de la Conferencia Internacional Americana, que pronto es rebautizada por la prensa y el público general como Conferencia Panamericana. Dice Smith que: "The concept of all the delegates working together to create a hemisphere of prosperous and democratic sisters nations reflected the popular view of Pan Americanism in the United States" (Smith 2000: 24). Bolívar andaba en el aire. Traducido.

A pesar de esas declaraciones de amistad y cooperación, los países latinoamericanos sospechaban de las intenciones de Estados Unidos. Se aproximaba la fecha de la reunión y muchos de los países invitados demoraban su respuesta. Smith explica que "Brazil would not confirm its conference attendance.

9 Una de las tareas de Curtis fue promover la causa de la conferencia. 
Peruvian officials . . . anticipated nothing of value to emerge from the meeting, and Chileans were openly suspicious of United States . . . few Argentines thought or cared much about the United States" (Smith 2000: 23). Finalmente, delegados de 17 países latinoamericanos aceptaron la invitación de Washington. No se harían presentes ni Haití ni República Dominicana y, los que acudieron, accedieron con reticencias y cada uno por sus propios motivos, especialmente con la idea de abrir oportunidades de negocio, pero siempre con la sospecha rondando ${ }^{10}$. José Martí, quien cubrió la Conferencia Panamericana para La Nación, de Buenos Aires, escribe sobre la reunión de Estados y alerta a los latinoamericanos:

Jamás hubo en América, de la Independencia acá, asunto que requiera más sensatez, ni obligue a más vigilancia, ni pida examen más claro y minucioso, que el convite de los Estados Unidos potentes, repletos de productos invendibles, y determinados a extender sus dominios en América . . . De la tiranía de España supo salvarse la América española . . . urge decir . . . que ha llegado la hora de declarar su segunda independencia (Martí 1991: 46).

Fue así, entre la retórica bolivariana y la amenaza del poder del "coloso del norte”, que los delegados latinoamericanos llegaron a Washington para asistir a la conferencia y para hacer, incluso antes de que comenzara la reunión internacional, el viaje en tren que el Departamento de Estado norteamericano había organizado para mostrar a sus invitados del sur el poder del norte. Comenzaba con ese recorrido la era dorada del panamericanismo y también una serie de intentos para establecer una comunicación más directa entre la América Latina y la anglosajona, un acercamiento de las lenguas y las mentes, que muchas veces no logró más que reforzar las imágenes fijas que una región tenía sobre la otra.

\section{Dependencias imperiales}

En la mañana del 3 de octubre de 1889 la lengua que más se hablaba en la estación Baltimore and Potomac, en Washington, era el español ${ }^{11}$. Varias decenas de personas se arremolinaban en el andén que flanqueaba a la locomotora

10 En las Obras completas de José Martí, quien cubrió la conferencia para La Nación de Buenos Aires, se registra así la explicación de la ausencia de los dos países caribeños: "Haití, como que el gobierno de Washington exige que le den en dominio la península estratégica de San Nicolás, no muestra deseos de enviar sus negros elocuentes a la conferencia de naciones; ni Santo Domingo ha aceptado el convite, porque dice que no puede venir a sentarse a la mesa de los que le piden a mano armada su bahía de Samaná” (Martí 1991: 33).

11 Ver Chicago Tribune (4 de octubre de 1889: 2). 
1053, una de las más poderosas fabricadas hasta el momento, y a los seis carros de lujo que la seguían ${ }^{12}$. En vez de las siglas que solían adornar a las locomotoras estadounidenses dando pistas sobre su lugar de origen, en los costados de la 1053 se desplegaba su marca de proveniencia con todas sus letras: Pennsylvania Rail Road. Este alardeo de fábrica, el lujo ferroviario nunca antes ensamblado sobre rieles norteamericanos, se debía a la calidad de los pasajeros que el tren transportaría durante seis semanas y casi seis mil millas: los delegados latinoamericanos invitados por el Departamento de Estado estadounidense a participar en la primera Conferencia Internacional Americana.

La idea del viaje, según las palabras de Curtis, encargado de los detalles prácticos y de la reunión posterior, era la de mostrarles a los invitados "the mills of New England, the forges and furnaces of Pennsylvania, the farms of the prairies, and the plantations of the South" (Smith 2000: 24) ${ }^{13}$. Es decir, el progreso, el acelerado crecimiento de los Estados Unidos, la grandeza que prometía. De ahí el tren y su locomotora, lo grande, lo poderoso, la máquina que se abre camino a todo vapor por el nuevo mundo. La minuta preparada por la compañía ferroviaria de Pennsylvania apenas acabado el viaje asegura que el tren destinado para la ocasión era "as complete in all its appointments as a metropolitan hotel or club-house” (Pennsylvania Railroad Co. 1889: 19). Al poder lo seguía la riqueza, el lujo. Después de la locomotora, el tren continuaba en seis carros: cuatro de ellos pomposos dormitorios con camarotes y literas, y, antes de la sucesión de coches-dormitorios, un coche-comedor y otro que servía de biblioteca, fumadero, salón de encuentros, barbería y que también contenía los baños. Dependencias imperiales, según lo llamaba la minuta del viaje. En este tren nada se desperdiciaba: uno de los carros llevaba un dínamo que se alimentaba con el vapor que producía el movimiento del tren, con éste se generaba la electricidad necesaria para iluminar cada compartimiento. El mismo vapor se usaba también para producir el calor que arroparía a los delegados en el viaje por el otoño estadounidense ${ }^{14}$.

12 Para más sobre la locomotora que realiza este viaje ver Fisher (1947).

13 Las plantaciones del Sur estaban reservadas para un segundo tour ferroviario que se haría después de la conferencia, y que tuvo que ser cancelado por falta de viajeros: solo dos delegados se inscribieron.

14 No todos los que participarían después en la conferencia se subirían al tren. Según cuenta el Chicago Tribune en su nota del 4 de octubre de 1889, los tres delegados de Argentina se abstuvieron inicialmente de participar en la excursión (aunque se unirán más tarde, como comentaremos después) como también lo hicieron uno de los tres delegados brasileños, dos de los tres de México, el único delegado de Nicaragua y uno de los tres funcionarios venezolanos. 
Este viaje que comienza bajo el signo de una traducción, de una re-escritura del panamericanismo acuñado por Bolívar (o de la escritura de una lectura), está marcado por grandes espacios de incomunicación. A bordo del tren no van traductores ni intérpretes, a pesar de que son pocos los latinoamericanos que hablan inglés y que ninguno de los delegados estadounidenses a bordo del ferrocarril domina el español ${ }^{15}$. Acá no hay sitio para la descontextualización de la traducción de la que habla Molloy porque lo que parece privilegiarse es el silencio entre las dos lenguas. Sin intérpretes ni traductores no hay posibilidad de ubicarse, el discurso traducido que hubiera hecho de puente entre los representantes de las dos regiones no llega a establecerse. De este modo, la contaminación, la relación que se da en toda traducción según dice Jorge Luis Borges, apenas se insinúa ${ }^{16}$. Aparece aquí y allá, aisladamente, sobre todo gracias a los pocos delegados latinoamericanos que manejan mejor el inglés -en calidad y en cantidad- que lo que sus contrapartes estadounidenses manejan el español. La comunicación, entonces, comienza quebrada. Los delegados no logran posicionarse del todo en ese lugar móvil al que se los destina apenas llegados a Estados Unidos y antes de comenzar siquiera las conversaciones sobre una posible unidad panamericana. Se podría suponer que esta falta de traductores e intérpretes se debió a un descuido del Departamento de Estado, un descuido relacionado con el poco conocimiento de los invitados latinoamericanos o con el etnocentrismo, como dice Smith, de los anfitriones.

A pesar de no contemplar el tema de la traducción, el Departamento de Estado quería asegurarse de que sus huéspedes fueran bien tratados y de que no se notara el esfuerzo detrás de tamaña empresa ${ }^{17}$. Por eso las tripulaciones de relevo, que tomaban el mando de la locomotora cuando el viaje debía seguir su curso por las noches (no todas las noches, algunas se pasaban en hoteles en la ruta). Por eso, también, el cigarro, el licor, la comida, los cuidados. La orden dada fue "Spare no expense" (Avisador Hispano-Americano 1890: 91), por lo menos en lo que respecta a los agasajos que los delegados recibieron en Chicago, pero lo cierto es que esa parece haber sido la orden detrás de todo el viaje. Atiborrar a los latinoamericanos con vistas, visitas a fábricas, ciudades, comidas, fiestas, hasta el punto del agotamiento, como veremos pronto. Llenarles los ojos de la grandeza del país antes de

15 Según mi investigación en los archivos un puñado de delegados (unos cinco) hablan un inglés fluido. Como se verá más adelante en este capítulo, mucho de los discursos que se dan durante el viaje son dados por los delegados en español y luego traducidos o reproducidos en parte por alguno de los delegados que habla inglés.

16 Ver Molloy (1998).

17 En el tren viajaban unas cien personas en total, entre tripulación, delegados, secretarios y prensa invitada. 
que se sentaran a discutir una moneda común para las Américas, la posibilidad de unidad aduanera y otros puntos de la agenda de la Conferencia Panamericana. Recordándoles, de vez en cuando, claro, sobre la idea bolivariana de la fraternidad entre los Estados americanos, aunque se hiciera visible la transformación, la manipulación, de las ideas detrás del Congreso de Panamá (que por cierto tampoco logró unir a los Estados de las otras Américas, que también guardaban sus propias tensiones entre sí). Uno de estos momentos se dio en la visita a una fábrica de ladrillos en Pittsburgh, la que reprodujo así el Avisador Hispano-Americano ${ }^{18}$ :

En compañía de nuestro siempre querido y respetado amigo don Nicanor Bolet Peraza recorrimos aquel vasto salón y tal vez nada llamó más nuestra atención que tender la vista hacia una columna de ladrillos, que se exhibían, y leer que en cada uno de ellos decía: Bolivar Fire Brick Company. - ¿El nombre de nuestro Libertador en ladrillos? -nos dijo Bolet Peraza, -Es caso curioso.” El encargado de la sección, que comprendió al momento nuestra natural curiosidad, tomó una de aquellas piezas de barro, y nos dijo: -"Bolivar brick, suitable for all purposes requiring ordinary heat resistance, and for fine outside work" (Avisador Hispano-Americano 1890: 91).

Bolet dijo enseguida: "Efectivamente, Bolívar resistió con fuego intenso a la tiranía, y la belleza de sus obras resplandece hoy en nuestras repúblicas. Está muy bien puesto el nombre a los ladrillos" (Avisador Hispano-Americano 1890: 91). Con esa explicación, el delegado traducía de vuelta, recuperaba a Bolívar, pero esa monetarización de la fraternidad panamericana, la torcedura del término, estaría presente durante todo el recorrido ferroviario. Seguramente los delegados venezolanos se hubieran sorprendido de saber que el pueblo aledaño a la fábrica de ladrillos también se llamaba Bolívar, nombre que habían adoptado luego de que el primer nombre elegido, Washington, fuera descartado por ya estar demasiado repetido a lo largo del país.

\section{All Aboard!}

El 4 de octubre Martí escribe (y traduce mientras lo hace):

18 El Avisador Hispano-Americano es un periódico de Nueva York dirigido por el cubano Enrique Trujillo y del cual José Martí fue frecuente colaborador. El Avisador no estaba entre la prensa acreditada para hacer el viaje en tren, pero cubrió el evento alcanzando a los viajeros en puntos clave del recorrido. Los artículos que se usan para este trabajo fueron recopilados en el libro de 1890 Relación del viaje de los señores delegados a diversos lugares de los Estados Unidos. 
Se abre el Herald y se lee: 'Es un tanto curiosa la idea de echar a andar en ferrocarril, para que vean cómo machacamos el hierro y hacemos zapatos, a veintisiete diplomáticos y hombres de marca, de países donde no se acaba de nacer'. . . El Tribune dice: 'ha llegado la hora de hacer sentir nuestra influencia en América: el aplauso de los delegados al discurso de Blaine fue una ovación'. . . Y el Sun dice: ‘Están vendidos a los ingleses estos sudamericanos que se le oponen a Blaine. . . El tren palacio ha comenzado, en tanto, a rodar en su camino de cinco mil cuatrocientas seis millas' (Martí 1994: 41).

Los delegados ya llevan un día de camino cuando Martí escribe lo que precede. Mientras la prensa local se pregunta por la sabiduría del viaje, por los motivos de los latinoamericanos, por el poderío de la nación anfitriona, el tren ya ha recorrido desde Washington a Nueva Jersey y desde ahí hasta la academia militar de West Point. El Chicago Tribune del mismo día en el que Martí se sienta a leer los artículos que cita toma un poco de distancia de las preguntas políticas que sobrevolaban el momento para contar lo que los viajeros vieron desde sus ventanas en ese primer tramo:

The day was clear and cool, a tinge of frost lingered in the air from over night. The maples had begun to turn scarlet and altogether the scene, familiar to the American eye, presented a charmingly novel effect to the great mass of the delegates who come from tropical and semi tropical climates and are accustomed to the sight of the spring cactus and the dark but ever green foliage of the South American jungles (Chicago Tribune 4 de octubre de 1889: 2).

La descripción de la naturaleza del norte es usada para marcar la diferencia entre los viajeros. Entre sus miradas. Unas, acostumbradas a los arces y, por extensión, al progreso, a las industrias que van alterando el paisaje natural. Otras, supuestamente, acostumbradas a las vistas de junglas y desiertos, que en este párrafo se transforman en el único territorio posible de Latinoamérica. Ellos y nosotros. América y el sur de América. Desde el primer día del relato de este viaje entran en juego los imaginarios, los estereotipos que se tienen de América Latina, de su paisaje, de la "raza" que la habita, de su naturaleza, sus gobiernos. Se sospecha, se especula. Muchos de los estadounidenses que reciben a los delegados en las distintas paradas del tren panamericano se preguntan cuál será el color de la piel de los viajeros, cómo sonarán, cómo podrán comunicarse, cuánto negocio traerán a los Estados Unidos. En una época en la cual los países industrializados organizaban exposiciones mundiales como forma de acercarse al resto del globo ${ }^{19}$, el tren funciona como una muestra en movimiento, una que corre en dos direcciones. Primero, sirve de gran exhibición móvil

19 De hecho, en los artículos sobre el viaje que publica el Chicago Tribune se menciona más de una vez la próxima Feria Mundial de Chicago de 1892. 
de Estados Unidos ${ }^{20}$. Los delegados latinoamericanos pueden mirar desde sus ventanas el país y lo que ofrece: sus campos, sus fundiciones, el crecimiento que parece imparable. Al mismo tiempo, es una exhibición en dirección opuesta. Los delegados están allí, adentro, y descienden una y otra vez de los seis carros del tren para ponerse ante la mirada estadounidense.

"Una inmensa concurrencia estaba repartida en los alrededores del paradero", dice El Avisador Hispano-Americano de la llegada de los delegados a Ann Harbor, y continúa:

La ciudad toda estaba de gala. Con gran trabajo pudieron los carruajes dirigirse a la Universidad de Michigan. Una gran procesión nos seguía . . . A la llegada frente al edificio donde está la universidad, encontramos a los 2,500 estudiantes que tiene ese establecimiento, formados en dos líneas, escoltando a la comitiva para penetrar en el salón de recepción (Avisador Hispano-Americano 1890: 53).

Los delegados, entonces, avanzaron por entre dos filas de 1,250 personas aproximadamente, que los miraban, atentamente, avanzar. Además, los vitoreaban. Dice el Chicago Tribune (que por cierto asegura que los estudiantes son 2000) que al paso de los latinoamericanos, los anfitriones gritaban: "Rah, rah, zing, boom, A-h-h-h!”. No hubo otro momento del viaje donde tantos ojos se posaran a la vez, y con la misma intensidad, sobre los viajeros, pero, de distintas maneras, la situación se repitió una y otra vez. Como si el juego de miradas fuera la manera de llegar a la formación de una imagen -que pasaba por confirmar o no estereotipos previos, ideas vagas- a su vez necesaria para sentarse después a la mesa de la negociación panamericana. El 21 de octubre de 1889, los delegados son agasajados en Chicago con una fiesta en la mansión de Potter Palmer, uno de los grandes hombres de negocio de la ciudad. Dice el Chicago Tribune del día siguiente:

In this illuminated exterior people from the neighboring side streets, and some even from distant parts of town, walked up and down or stood in groups to get such glimpses as they could of the arriving Spanish-Americans and the captivating scenes within. As the first carriages, which contained the delegates, began to arrive, the crowd gathered on the sidewalk opposite the doorway. A subdued but ecstatic murmur went up as the doors opened to admit Sr. Juan P. Velando of Bolivia, and his escort, and revealed for a moment the imposing circular hallway of the house, with its ring of marble pillars (Chicago Tribune 22 de octubre de 1889: 2).

Así como los delegados son observados atentamente, desde cerca, por quienes los reciben y agasajan, son también vistos desde lejos, al pasar, por las multitudes de gente común que quieren acercarse al extranjero y, también, a la pompa

20 Le debo a Sylvia Molloy esta idea. 
que rodea el viaje. Porque la riqueza de América no es para todos y el lujo que el tren del progreso ha ido llevando al Nuevo Mundo se disfruta a puertas cerradas, por unos pocos. Las miradas de la multitud entonces se posan sobre esas dos extranjerías: la riqueza y la latinoamericanidad de los delegados, quienes tal vez traigan aun más riqueza, $y$, tal vez, por qué no, podría ser la pregunta de esas multitudes, para todos. Esa podría ser una forma de entender el revuelo que los latinoamericanos van creando a su paso. El Chicago Tribune del 1 de noviembre cuenta que:

\footnotetext{
It's in the smaller cities that the outpouring of the people to receive the travelers is most noticeable. Every little station platform by which the train rushes is lined with its crowd of country people shouting their welcome and waving an on-speeding. Bands of music are at many of the stations and people waving flags of all the nations of the three Americas are seen as the train flashes by . . . (this) is a source of much satisfaction to the southerners (Chicago Tribune 1 de noviembre de 1889: 7).
}

Las miradas aparecen incluso cuando el tren avanza veloz, sin detenerse. Y cuando se detiene, cuando los delegados bajan del tren-exhibición y se entregan a los ojos anfitriones, las miradas se muestran distintas en su calidad. Por ejemplo, en Portland, Maine, donde llegan los viajeros cinco días después de haber comenzado el tour. Después de dejar la estación, los delegados son llevados a recorrer la ciudad en carruajes. Dice en otro momento el diario de Chicago: "The citizens were prepared for the visitors and flags floated from many buildings; almost every window on the route was filled with faces, hats were raised and the children turned out in numbers" (Chicago Tribune 9 de octubre de 1889: 2).

Los niños salen afuera, corren, juegan junto al paso de los delegados. Además, se levantan sombreros. Hay gente en la calle que intenta ver a los delegados desde las veredas, se asume, pero también hay muchos que se parapetan desde las ventanas. Quieren ver, pero no participar del todo. Mirar un poco, golpear el vidrio o abrirlo para ver mejor la cara fugaz de los latinoamericanos. Así se hace presente en este viaje la mirada curiosa, que no se compromete pero observa, anota, especula. Los delegados latinoamericanos también hacen un ejercicio de la mirada durante los 42 días de tour ferroviario. Observan el paisaje vasto que cambia mientras el tren se mueve. Observan cómo se fabrican ladrillos, armas, ropa, los bienes de Estados Unidos, exhibidos ahora como productos terminados especialmente para ellos, como cuenta el Tribune:

After looking over the factories the party was escorted to a new mill, where had been arranged a display of textile and other goods produced in Manchester in the half of a working day. It made a most formidable showing in its extent, and was, moreover, arranged so as to make a most attractive exhibition . . . When the exhibit had been duly admired 
the party were seated at a luncheon furnished by the citizens. . . (el énfasis es mío, Chicago Tribune 9 de octubre de 1889: 2).

Los delegados no solo miran lo que está frente ellos, sino lo que ha sido arreglado para ellos. Pueblos, ciudades y fábricas despliegan performances para los visitantes. Se adornan y se presentan en la manera en que quieren ser vistos por estos latinoamericanos que tal vez, y ese es uno de los motivos y de las posibilidades que se plantean con este viaje, terminen siendo parte de la misma América, de la Pan América. "El otro" que se vuelve vecino, un conocido, ya no tan lejano. Algunos de los latinoamericanos también llegan a percibir la familiaridad que los rodea, por lo menos eso le ocurre al reportero de El Avisador, que va en el tren de los delegados en el tramo de Pittsburg a Filadelfia:

Verdaderamente, nunca habíamos visto a los campos de Estados Unidos representados con tanta grandeza . . . Hay panoramas que reflejan la hermosura de los campos de México, la belleza de los de Cuba . . . La herradura que forma la línea férrea, para vadear un río y una cuesta, es de gran efecto. La roca viva que se ha perforado por la mano del hombre, para hacer aquella obra, es admirable (El Avisador Hispano-Americano 1890: 91).

El reportero "reconoce" la tierra como propia o parecida a la propia. De esa manera los triunfos del progreso se sienten menos extranjeros, más cercanos. En este viaje, Estados Unidos presenta sus riquezas, su creciente dominio sobre la naturaleza, su progreso extraordinario: el producto de "solo" medio día de trabajo en Manchester, el poder del gas natural en Pittsburg, tres mil luces eléctricas encendidas a la vez por un dínamo y muchos ejemplos más por el estilo. Pero también se les muestra algo más a los delegados. El primer día de viaje, como ya se mencionó, termina en la academia militar de West Point, con un desfile militar y con el descubrimiento de los bustos de tres generales estadounidenses. El tercer día se destina para la visita a una correccional de Boston, ubicada en la isla Deer, donde los viajeros son recibidos por unos 300 chicos detenidos en la correccional quienes les cantan canciones vestidos con uniformes azules y guantes blancos. Los organizadores del viaje no son los primeros en incluir cárceles como parte de los tours para mostrar el progreso en Estados Unidos. Tocqueville mismo había visitado el Eastern State Penitentiary de Filadelfia como parte del viaje del que nacerá Democracy in America. Después, cuando el viaje llegaba a las dos semanas, los delegados ya exhaustos con el ritmo frenético del tour por los Estados del norte, son llevados a un asilo mental. Ya la vista está cansada, como lo insinúa el mismo Chicago Tribune:

Without a chance of getting to a hotel and declining further attentions they were taken to Newburg. There they made the tour of the insane asylum, dragging themselves up countless flights of stairs only to look upon the saddest thing in the universe -faces robbed of 
the light of reason. After this they were taken through mill after mill, all extremely interesting, but to most of the visitors a tremendous bore (el énfasis es mío, Chicago Tribune 16 de octubre de 1889: 2).

Los delegados se quejan de la poca flexibilidad del itinerario planeado para ellos, pero el viaje del tren ya está planificado y la máquina no se detiene. Están obligados a mirar y estas últimas tres paradas de las que hablamos les muestran que el progreso no se logra sin dejar residuos y sin la autoridad ni la disciplina para disponer de ellos, reeducarlos, transformarlos en algo útil, aunque esa utilidad no sea más que una performance. De esta manera, sin quererlo, estas paradas se presentan como advertencias del progreso y, también, como amenazas. Estados Unidos es un tren que avanza rompiendo cosas a su paso, de las que sabe disponer. Ese es el naciente modelo que se exhibe ante las ventanas de un tren en movimiento, cargado de latinoamericanos que pronto se sentarán a una mesa a decidir la consolidación o no de un hemisferio tutelado por Estados Unidos.

\section{Miradas sobre la piel}

En su artículo sobre Pedro Henríquez Ureña y el latinoamericanismo Fernando Degiovanni menciona una encuesta hecha por la Oficina de Asuntos Interamericanos de Estados Unidos en 1941 sobre "What people in the United States think and know about Latin America and Latin Americans". El cuestionario listaba una serie de adjetivos descriptivos y pedía que quienes contestaran eligieran las palabras que representaban mejor a los habitantes de Sudamérica y Centro América. Dice Degiovanni (quien toma esta información del trabajo de Lars Shoultz sobre las políticas de Estados Unidos hacia Latinoamérica):

Los resultados eran del todo desalentadores para una política del entendimiento: setenta y siete por ciento de los encuestados -la mayoría- respondían que los latinoamericanos eran "dark skinned", y los atributos que seguían en su caracterización eran "quick-tempered", “emotional”, "backward”, "religious”, "lazy”, “ignorant” y "suspicious”. El adjetivo menos elegido era "efficient” (Degiovanni 2015: 145).

¿Cómo se había llegado en Estados Unidos a tener estas ideas sobre Latinoamérica y sus habitantes? Dice Degiovanni que Henríquez Ureña entiende que "esas representaciones son el producto de años de construcción de un discurso público a partir de la teoría positivista de los "caracteres nacionales". Incluso en Latinoamérica misma, esas ideas sobre las razas estaban particularmente en 
boga para los años de la Primera Conferencia Panamericana ${ }^{21}$. También son parte de las expectativas de la gente que recibía a los delegados latinoamericanos en su recorrido ferroviario, en lo que esperaban de sus costumbres, de sus apariencias. Un ejemplo de la fuerza de estos estereotipos en formación está en esta escena descrita por el Chicago Tribune, donde se da cuenta de la llegada de los delegados a la ciudad de Chicago y de lo que pasa cuando son "vistos" por primera vez por quienes los esperan:

Much surprise was manifested when the delegates were seen. A vague impression seemed to prevail among the better acquainted persons in the crowd that a swarthy lot of gentlemen, a shade lighter than octoroons and with the habit of rolling cigarettes, would appear and the more densely ignorant fostered a notion of persons with wide sombreros and trousers with lambrequins on them. But when they came, a meeting of American bank directors might not have been distinguished from the crowd on the parlor floor of the hotel -all natty, all not swarthy, but darkly pale, all dressed in the fashion, and mostly all speaking English perfectly ${ }^{22}$ (el énfasis es mío, Chicago Tribune 20 de octubre de 1889: 1).

Lo primero que hace aquí el Tribune es dividir al grupo que espera a los delegados según su conocimiento sobre lo que es "ser latinoamericano" o, mejor dicho, sobre el conocimiento respecto a cómo "debería verse" un latinoamericano ${ }^{23}$. Sylvia Molloy dice que las lecturas que el primer mundo hace del tercer mundo siempre son reduccionistas, ansiosas ${ }^{24}$. La imagen de Latinoamérica que el periódico adjudica a los menos duchos en las apariencias de la región evoca una particular mezcla de mexicanos de sombreros grandes y gauchos de Rugendas, es decir, hombres vestidos para la vida afuera, la vida bajo un sol inclemente, la de lacear caballos, la de dormir siestas apoyados en rocas o troncos. Por otro lado, se le asigna a los más informados la idea de que la principal diferencia con los delegados estaría en el color de la piel, que en los visitantes sería más oscura. Esta idea se hace presente otras veces a lo largo del viaje, como en esta visita a las empaquetadoras de carne de las afueras de Chicago:

It was so chilly that one gentleman was moved to ask a dark-complexioned reporter if he did not find the climate rather severe up here. The reporter said he did at first, but after staying here four or five winters he had gotten sort of used to it. The gentlemen walked away and made no more attempts to pick out delegates by their appearances. In fact, that

21 Ver Reid (1977: 120).

22 Como se dijo anteriormente, en realidad unos cinco delegados latinoamericanos hablaban el inglés de manera fluida.

23 La mirada sobre la región era una mirada general, de bloque, que no hacía mayor distinción entre los países que la conformaban, con la excepción -que tampoco admitía mucho detalle a nivel de conocimiento socio-cultural- de los llamados países ABC: Argentina, Brasil y Chile.

24 Ver Molloy (2005). 
was an extremely difficult thing to do. Mix them up with the Reception Committee and with a few exceptions one could not tell who was a delegate and who was not (Chicago Tribune 22 de octubre de 1889: 1).

En el título de la nota, otra vez la referencia a los ojos, la mirada, el verse. La semejanza entre unos y otros, ellos y nosotros, confunde a los estadounidenses que no han conocido antes a gente de Latinoamérica. La distinción que esperan pasa por el color de la piel, la ropa, las costumbres, la lengua. Y el encuentro con personas "all natty, all not swarthy, but darkly pale, all dressed in the fashion, and mostly all speaking English perfectly" (Chicago Tribune 22 de octubre de 1889: 1) es más desconcertante que la diferencia radical augurada ${ }^{25}$. ¿Cómo manejar esa familiaridad con ese otro al que se suponía tan lejano? Una forma es precisamente leerlo en términos familiares, con los códigos del mundo del estadounidense de fines del siglo diecinueve, como en este fragmento que pertenece al artículo del Tribune del 20 de octubre:

The crowd of 500 or 600 persons ... surged forward to see Major Domo William Elroy Curtis ... reappear with great radiance escorting a tall man with a pointed grey beard who look like an Englishman out here to shoot buffalo. This was Dr. Alberto Nin of Uruguay, who speaks English with an English accent and looks less like a Spaniard than Lieut. Read of the army, who had his other arm (el énfasis es mío, Chicago Tribune 20 de octubre de 1889: 1).

Los delegados van siendo rápidamente reapropiados por la narrativa "americana”. Van siendo traducidos, borgianamente contaminados. En este caso, Nin es puesto junto a los ingleses, por su apariencia y por su acento y perfecto inglés. También, en esa misma dirección, acercándolo, se dice que se ve menos "Spaniard” que el teniente estadounidense que le toma de un brazo. El delegado uruguayo aparece entonces revestido con los ropajes de un inglés, al mismo tiempo, la imagen que se le asigna trae a la cabeza la de los hombres que solo 20 años antes del viaje panamericano habían atravesado las grandes praderas estadounidenses, disparando desde las ventanas de un tren en marcha hasta casi extinguir a los búfalos y, con ellos, a los indígenas norteamericanos. Es decir, el delegado también es inscrito en esa narrativa de conquistadores de la tierra norteamericana tal vez augurando una nueva era de bonanza para el país gracias a los posibles tratos panamericanos. Además, las imágenes a las que se recurre en las citas anteriores recodifican a los delegados colocándolos no solo en un terreno familiar sino asignándoles un lugar específico en este: el de la riqueza, el

25 Es interesante notar cómo se generaliza el dominio del inglés de los delegados, ya que son mayoría los que no hablan inglés fluidamente. Sin embargo, el puñado que sí maneja la lengua a la perfección pasan a representar a todo el grupo. 
del poder. Se los descontextualiza y se les coloca en un lugar nuevo que obedece a las expectativas estadounidenses sobre el panamericanismo. Esto es lo que se hace al decir que es difícil distinguirlos de los miembros del comité de recepción o lo difícil que sería separarlos de una reunión de banqueros estadounidenses ${ }^{26}$. Este tipo de comparaciones viene a alimentar la idea de los beneficios comerciales que podría traer la unidad panamericana a Estados Unidos.

Como ya se ha visto, las imágenes que se tienen sobre los delegados y por extensión sobre Latinoamérica cubren un espectro amplio que va desde la diferencia racial, que comprende la idea de la inferioridad racial de América Latina, hasta la formulación de los delegados como sujetos con poder económico y político. De hecho, para algunas voces de la época, como demuestra este fragmento de un artículo publicado por The Nation el 17 de octubre, ese mismo imaginario, ese espacio de conocimiento insuficiente que se completa con especulación y estereotipos, habría estado en el origen de la invitación al tour ferroviario:

It may be said that its planning showed other motives than delicate consideration for the nation's guest. Most of them are men of mature years, for whom six weeks of life in railroad cars, in a changeable climate, is not exactly a luxury. Besides, there is in the thing almost an implication against their intelligence -as it would be a good thing to treat them as the Government used to treat the Sioux chiefs Red Cloud and Crazy Horse- take them to Washington, show them the sights, and so impress them with Uncle Sam's power that they would see no hope in opposing him. These delegates are traveled gentlemen, who are not easily awe-stricken (el énfasis es mío, Smith 2000: 25).

El artículo de The Nation es muy claro en su crítica. Habla, como dice Smith, del etnocentrismo y la inexperiencia de Blaine y de Curtis (recordemos, las principales mentes detrás de la Conferencia Panamericana), de todo lo que se ignora sobre Latinoamérica, lo poco que se sabe también de sus elites intelectuales y sus gobernantes. En este espacio de desconocimiento es posible construir una imagen de los delegados que amerite un trato del Departamento de Estado equivalente al recibido por los jefes Sioux en su momento. Se les ofrece un tren con todas las comodidades posibles para partir a un viaje absolutamente demandante, plagado con actividades hasta el último minuto. Las comodidades y lujos que se les ofrecen a los viajeros pueden leerse bien como una forma de impresionar o de presionar una colaboración con una unión hemisférica liderada por Estados Unidos. Todas esas visitas a fábricas, molinos, mataderos, ciudades en crecimiento, la academia militar, la correccional, el asilo mental, pueden leerse como un tour dedicado a

26 En cada pueblo o ciudad a la que llegaban los delegados, los esperaba un comité de recepción formado por los honorables del lugar, ya sea industriales, dueños de tierras, alcaldes o semejantes. 
promover las maravillas de un país en plena expansión, pero también como una velada amenaza con respecto al poderío en construcción del coloso del norte.

Por su parte, los delegados latinoamericanos observaban y tomaban nota. Como ya se dijo antes, muchos de ellos habían aceptado la invitación a regañadientes y por diversos motivos ${ }^{27}$. En general, los países de América Latina desconfiaban de las intenciones de Estados Unidos. Sin embargo, a pesar de la desconfianza, los delegados se entregaron al tour ferroviario con bastante entusiasmo, aunque a poco andar el horario repleto de actividades comenzó a pesarles. La prensa que cubría el viaje dio cuenta del cansancio de los viajeros y del sinsentido del cúmulo de actividades al que se les sometía. Un día antes de que se publicara la nota de The Nation citada anteriormente, el Chicago Tribune dedicó a los latinoamericanos un artículo donde se comentaba sobre esto:

It is a weary, weary party of South Americans . . . They have been whirled over states till they have forgotten the number . . . They have been banqueted till their stomachs are as weary as their heads ... The early rising feature of the present expedition is the most serious consideration to the South Americans. One of their number explained, in speaking of the fatigues of the journey, that the southerners were in the habit of rising at easy hours of the forenoon, of eating when they feel disposed, and no-customed [sic] to having railways trains await their deliberated will (Chicago Tribune 16 de octubre de 1889: 2).

Mientras el viaje se desarrolla algunos de los delegados comienzan a demostrar su cansancio. Algunos abandonan la expedición en ciertos puntos, para retomarla en otros, algunos se niegan a levantarse por la madrugada. Otros, como el que habla con el Tribune, se deciden a proclamar las diferencias culturales que hacen del viaje una experiencia agotadora, pero al mismo tiempo toman nota de lo que van viendo. Como dice el periódico de Chicago del 20 de octubre, que reproduce una conversación en la que participan tres delegados que manejan el inglés. En ella participan el alcalde de Chicago y otros funcionarios estadounidenses, el delegado Horacio Guzmán de Nicaragua (el con mejor nivel de inglés, según el periódico), el delegado de Perú F.C.C Zegarra ("a typical Spaniard from Peru”) y el delegado argentino Manuel Quintana. Todos se muestran muy contentos con lo que han visto en la primera mitad del viaje. Zegarra anuncia que ha visto "más glorias" de las que pudo haber soñado alguna vez, entonces lo interrumpe Quintana, quien dice que aún faltan más glorias, como los “stockyards" y las "packing houses" de los mataderos. "If we get from them

27 Dice Smith: "Brazil was rumored to be eager for a commercial treaty with the United States to boost its declining sugar industry, Chileans hoped for regular steamship traffic to the United States and supported a common silver currency for the hemisphere. At the same time, most countries saw the conference as an opportunity to air a variety of grievances and initiatives. . ." (Smith 2000: 23). 
new ideas we will have filled our missions" (Chicago Tribune 20 de octubre de 1889: 1), dice uno de los representantes de Argentina, país que no tiene mayor interés en la conferencia misma.

\section{La lengua}

Este encuentro de las regiones también fue un encuentro entre lenguas, más bien un desencuentro, como ya hemos sugerido. La noche antes de embarcarse en el viaje los delegados fueron agasajados en el hotel donde vivía Blaine a la espera de que se terminara la construcción de su casa. Antes de la cena, se había reunido el pleno de la conferencia para dar el discurso inaugural de la reunión que sería retomada después de las seis semanas de tour ferroviario. Los discursos se dieron solo en inglés. No había intérpretes, tampoco traductores. Estados Unidos marcaba su afán de liderazgo hemisférico estableciendo también una jerarquía entre las lenguas. La relación de poder entre las culturas en juego se explicitaba también en el orden de la palabra. Este desequilibrio se desplegaría en el viaje en tren y luego se corregiría parcialmente, de manera imperfecta, en el resto de la Conferencia Panamericana ${ }^{28}$. Pero en ese tren, en ese espacio cerrado, móvil, que lleva y trae a todos los delegados, el desencuentro se plasma en silencios, en la torpeza de la incomunicación.

Aunque son pocos los delegados latinoamericanos que manejan el inglés, son aun menos, apenas un par, los estadounidenses que hablan algo de castellano. Esto hace que las intervenciones de los invitados al viaje sean, en general, muy pensadas porque como dice el periódico de Chicago, "impromptu interpretation is not an easy task" (Chicago Tribune 22 de octubre de 1889: 2). Continúa el diario: "At the banquets the foreigners usually decline to trust themselves before an audience trammelled with a foreign idiom, and they speak in their own language”. Las únicas veces que un delegado latinoamericano se aventura a hablar en inglés, dice el Tribune, es cuando el discurso se ha escrito la noche anterior y ha sido traducido por alguno de los secretarios de la delegación correspondiente. "But when the talk is extemporaneous the poor secretary must have his wits

28 El Chicago Tribune explica que en la conferencia se usaría inglés y español (es de suponer que los delegados brasileños usarían el portugués, aunque no se hace mención de esto en el artículo). Luego un intérprete haría un resumen de lo dicho y al día siguiente se publicarían versiones completas y bilingües de las intervenciones de los delegados para permitir reacciones a estas mismas. Al parecer esto no se respetó del todo. Según Smith, durante la conferencia "there were no English-to-Spanish translations made of opening conferences speeches" (Smith 2000: 24). 
about him . . . they usually succeed remarkably well in doing this" (Chicago Tribune 16 de octubre de 1889: 2).

Es de suponer que el discurso que dio el delegado de Venezuela Nicanor Bolet Peraza a poco andar de la expedición, y que acá cito de acuerdo a la versión en español que publicó el Avisador Hispano-Americano, fue uno de estos traducidos por un secretario:

Es la primera vez que hablo en público en un idioma extraño, el cual he aprendido para expresar mis necesidades y no para expresar mis sentimientos (Aplausos) . . . Yo apelo, pues, a la indulgencia del respetable auditorio. Yo suplico a mis oyentes, que si no pueden entenderme bien, tomen en su mano el diccionario del corazón, y lo abran por la página de la Fraternidad (Aplausos) (El Avisador Hispano-Americano 1890: 19).

Con sus palabras Bolet Peraza califica al inglés como lengua utilitaria, para hacer transacciones, trámites, a diferencia del francés que, en esos años, era para las elites latinoamericanas el idioma del conocimiento y de las letras. Otra cosa que hace el venezolano aquí es apelar a una lengua común para el hemisferio, una que no tiene que ver con reglas gramaticales ni lingüísticas, una lengua de la fraternidad que en la realidad (ni en el viaje en tren ni en la conferencia que lo sigue) no se sostiene. A veces se intenta, como cuando en una de las múltiples paradas del tren el delegado chileno José Alfonso hace un apasionado discurso en español que es vitoreado por sus pares hispanos y, acto seguido, por los estadounidenses que no entienden una palabra de lo que dice pero que se contagian con la emoción del momento. Pero ese contagio es momentáneo. No es contaminación. En general, lo que abunda en el viaje son los desencuentros, los pedidos de traducciones improvisadas, los esfuerzos de algunos por acercarse a los otros. Hay muchos momentos de frustración lingüística, algunos provocados por la ignorancia del idioma ajeno y, aunque suene extraño, otros provocados por el conocimiento del mismo. En el relato de la llegada a Chicago que hace el Tribune se da una curiosa mezcla de estos dos últimos. El periódico cuenta que misteriosamente nadie de entre la multitud que esperaba en la estación escuchó venir el tren. Los oídos se cerraron ante la llegada del vapor que debe haber sonado alto y la llegada súbita de la locomotora y de sus seis carros causó una pequeña conmoción que desbandó el orden previsto para la llegada de los viajeros. Rápidamente se formaron dos filas de policía en una de las salidas del tren para facilitar la bajada de los delegados y su encuentro con el comité de recepción que intentaba acercarse a ellos.

Al caos de la llegada lo sucedió el desencuentro de las lenguas. "The members of the committee hastened to the different coaches to welcome the guests, and -well, it is best to say nothing of those who met people who could speak nothing but Spanish" (Chicago Tribune 20 de octubre de 1889: 1). Sobre el mur- 
mullo feroz de la multitud se escuchaban aquí y allá las voces que llamaban a los latinoamericanos desde los carruajes que los llevarían al hotel asignado para ese tramo del viaje. De una manera u otra, la comitiva logró salir de la estación y llegar al hotel. Allí, entre quienes los esperan estaba un Coronel Sam Parker, quien llevaba en un bolsillo de la chaqueta un diccionario de español, el que había estado mirando durante la mañana. Cuando entraron los delegados, Parker se acercó a uno de ellos y saludó:

"Seenyore," began Mr. Parker.

"Call me mister," said the visitor, "and kindly ask what you call 'front' to show me to my room, for I am tired."

Col. Parker sank to the floor exhausted (Chicago Tribune 20 de octubre de 1889: 1).

Al parecer, los invitados latinoamericanos con conocimientos del inglés no siempre fueron atentos a los esfuerzos estadounidenses por hablar español. Aunque también cabe preguntarse por el rápido desánimo de los estadounidenses, sobre todo si se tiene en cuenta que el número de los delegados latinoamericanos que hablaba español era limitado.

Just as the train got under way one of the gentlemen informed a delegate that they were bound for the establishment of "Armour y Cia. Saladeros y Comisionistas de Provisiones y Propietarios de Armour Glue Works."

"O, yes”, said the delegate, “Going to Armour's. I've heard of him.”

Then the man went off and sat down by himself, and tore up a card that Armour had gotten out in Spanish, stating that his "oficino" [sic] was at No. 205 Calle de La Salle (Chicago Tribune 22 de octubre de 1889: 1).

Tal vez la actitud de los latinoamericanos haya sido motivada por el cansancio y el enojo producido por el ya largo viaje y el feroz ritmo impuesto por los organizadores del mismo. O tal vez haya sido el cansancio de enfrentarse a un español que no lograba llegar a ser tal, como cuenta el Tribune casi al final del periplo.

The appointment of the local reception committees in the various cities brings out many amateur linguists. St. Louis was specially blessed with these. They seemed to think the visitors spoke little English, and committeemen have all day been springing Spanish sentences from hidden phrasebooks. Occasionally an especially enterprising entertainer would be seen who had covered his cuff with convenient phrases and was thereby able to astonish his friends as well as the visitors. The trouble with this kind of Spanish is that it is unintelligible to an American coming from anywhere between Patagonia and Hudson's Bay (el énfasis es mío, Chicago Tribune 30 de octubre de 1889: 3).

El periódico reconoce aquí la inutilidad del español balbuceado, de las frases inconexas que se prestan más para la entretención de los invitados que para la comunicación. La lengua como circo no sirve para lograr un hemisferio unido, como el que aparece aquí, ese que va desde la Patagonia hasta la bahía del 
Hudson y que se une en la incapacidad de entender un idioma del que se sacan y repiten frases sin sentido. Ya se ha visto que entre las elites de Estados Unidos y de los países latinoamericanos hay un desequilibrio evidente sobre el conocimiento del otro, sobre la lengua del otro. Aquí no hay traducciones sino asaltos a la lengua extraña. Estados Unidos vuelca su mirada sobre los vecinos del sur sin sacar sus ojos de su propio idioma, de su desarrollo imparable, de su autootorgado excepcionalismo, de sus expectativas. Es decir, mira hacia allá sin ver más que lo superficial o sin poder despegar esa mirada de la imagen de Latinoamérica que él mismo se ha creado.

The shocking ignorance of the people everywhere in this country as to everything pertaining to the home countries of the visitors causes much well-bred surprise. . . 'We have at Buenos Ayres' said a delegate from the Argentine Republic, 'a newspaper with a greater circulation than any other in the Western Hemisphere -including your United States, mind you- and even with that evidence of civilization it is hard sometimes to convince our friends here that we live in anything but a country of savages (Chicago Tribune $22 \mathrm{de}$ octubre de 1889: 1).

La mirada estadounidense no puede dejar de mirar a través de los estereotipos y de las ideas pre-formadas, como lo demuestra esta pequeña escena con la que se describe en el Chicago Tribune la visita que hacen los delegados a una granja de caballos de carrera:

“Beautiful! Superb! Perfect!” The stock of English adjectives was exhausted, and the distinguished representatives of all America called their native Spanish to their aid to express their admiration of the sight. "Magnífico! Hermosa! Splendito! Spectaculo linda!” (el énfasis es mío, Chicago Tribune 23 de octubre de 1889: 1).

Estos errores del periódico pueden parecer pequeños pero son tan grandes como la idea de que no se necesita corroborar el habla que se desconoce. No se llega a tomar en serio a esta lengua ajena, que se sigue presentando pintoresca, idioma de retablo, la menos poderosa en el juego de jerarquías que se está recién estableciendo entre las distintas regiones de América. Este lugar de desencuentro entre las lenguas involucradas en el intento de construir una Pan-América se vuelve el espacio necesario para la aparición de traductores, intelectuales y escritores que puedan establecer un puente $\mathrm{u}$ otra forma de negociación de los poderes atribuidos a las lenguas y culturas en juego. Serán ellos los que desafiarán los imaginarios anquilosados sobre Latinoamérica mientras tienden redes con sus pares de la región, entablando una conversación que irá más allá de las modas que acompañan a ciertos eventos como la misma conferencia de la que hemos hablado. Porque después de esta, que en concreto no logró nada más importante que la creación de una oficina de comercio para las repúblicas americanas, que luego se convertiría en la Unión Panamericana, el español y lo panamericano gozó de 
cierta popularidad en Estados Unidos. Las clases de español se multiplicaron en escuelas y universidades, la oficina de comercio no paró de imprimir catálogos, guías de viaje, textos con los que conocer más a los vecinos del sur. El problema es que la fascinación parece haber sido unidireccional, además de breve, como se verá en los capítulos siguientes. Latinoamérica seguía alimentando sus sospechas sobre las intenciones de Estados Unidos. Así lo decían sus políticos, sus intelectuales. Así lo escribirían Darío y Rodó con sus Arieles y Calibanes. Y cómo no nutrir la sospecha, si vendría la intervención en Cuba que se convertiría en guerra contra España, la apertura del Canal de Panamá, si después de la Primera Guerra Mundial y el reordenamiento geopolítico subsiguiente serían solo unos pocos los que continuarían mirando a Latinoamérica. Entre ellos, los traductores e intelectuales que exploraremos más adelante, quienes siguieron, a pesar de todo, tratando de conectarse con esas mentes pares de los países de Latinoamérica. 